\title{
COFINITENESS AND COASSOCIATED PRIMES OF LOCAL COHOMOLOGY MODULES
}

\author{
MOHARRAM AGHAPOURNAHR and LEIF MELKERSSON
}

\begin{abstract}
Let $R$ be a noetherian ring, $a$ an ideal of $R$ such that $\operatorname{dim} R / \mathfrak{a}=1$ and $M$ a finite $R$-module. We will study cofiniteness and some other properties of the local cohomology modules $\mathrm{H}_{\mathfrak{a}}^{i}(M)$. For an arbitrary ideal $a$ and an $R$-module $M$ (not necessarily finite), we will characterize $a$-cofinite artinian local cohomology modules. Certain sets of coassociated primes of top local cohomology modules over local rings are characterized.
\end{abstract}

\section{Introduction}

Throughout $R$ is a commutative noetherian ring. By a finite module we mean a finitely generated module. For basic facts about commutative algebra see [3] and [9] and for local cohomology we refer to [2].

Grothendieck [7] made the following conjecture:

CONJeCture. For every ideal a and every finite $R$-module $M$, the module $\operatorname{Hom}_{R}\left(R / \mathfrak{a}, \mathrm{H}_{\mathfrak{a}}^{n}(M)\right)$ is finite for all $n$.

Hartshorne [8] showed that this is false in general. However, he defined an $R$-module $M$ to be $\mathfrak{a}$-cofinite if $\operatorname{Supp}_{R}(M) \subset \mathrm{V}(\mathfrak{a})$ and $\operatorname{Ext}_{R}^{i}(R / \mathfrak{a}, M)$ is finite (finitely generated) for each $i$ and he asked the following question:

Question. If $a$ is an ideal of $R$ and $M$ is a finite $R$-module. When is $\operatorname{Ext}_{R}^{i}\left(R / \mathfrak{a}, \mathrm{H}_{\mathfrak{a}}^{j}(M)\right)$ finite for every $i$ and $j$ ?

Hartshorne [8] showed that if $(R, \mathfrak{m})$ is a complete regular local ring and $M$ a finite $R$-module, then $\mathrm{H}_{\mathfrak{a}}^{i}(M)$ is a-cofinite in two cases:

(a) If $a$ is a nonzero principal ideal, and

(b) If $\mathfrak{a}$ is a prime ideal with $\operatorname{dim} R / \mathfrak{a}=1$.

Yoshida [14] and Delfino and Marley [4] extended (b) to all dimension one ideals $\mathfrak{a}$ of an arbitrary local ring $R$.

In Corollary 2.3, we give a characterization of the a-cofiniteness of these local cohomology modules when $\mathfrak{a}$ is a one-dimensional ideal in a non-local 
ring. In this situation we also prove in Theorem 2.7, that these local cohohomology modules always belong to a class introduced by Zöschinger in [16].

Our main result in this paper is Theorem 2.10, where we for an arbitrary ideal $\mathfrak{a}$ and an $R$-module $M$ (not necessarily finite), characterize the artinian a-cofinite local cohomology modules (in the range $i<n$ ). With the additional assumption that $M$ is finitely generated, the characterization is also given by the existence of certain filter-regular sequences.

The second author has in [10, Theorem 5.5] previously characterized artinian local cohomology modules (in the same range). In case the module $M$ is not supposed to be finite, the two notions differ. For example let a be an ideal of a local ring $R$, such that $\operatorname{dim}(R / \mathfrak{a})>0$ and let $M$ be the injective hull of the residue field of $R$. The module $\mathrm{H}_{\mathfrak{a}}^{0}(M)$, which is equal to $M$, is artinian. However it is not $\mathfrak{a}$-cofinite, since $0 \underset{\dot{M}}{\dot{a}} \mathfrak{a}$ does not have finite length.

An $R$-module $M$ has finite Goldie dimension if $M$ contains no infinite direct sum of submodules. For a commutative noetherian ring this can be expressed in two other ways, namely that the injective hull $\mathrm{E}(M)$ of $M$ decomposes as a finite direct sum of indecomposable injective modules or that $M$ is an essential extension of a finite submodule.

A prime ideal $\mathfrak{p}$ is said to be coassociated to $M$ if $\mathfrak{p}=\operatorname{Ann}_{R}(M / N)$ for some $N \subset M$ such that $M / N$ is artinian and is said to be attached to $M$ if $\mathfrak{p}=\operatorname{Ann}_{R}(M / N)$ for some arbitrary submodule $N$ of $M$, equivalently $\mathfrak{p}=\operatorname{Ann}_{R}(M / \mathfrak{p} M)$. The set of these prime ideals are denoted by $\operatorname{Coass}_{R}(M)$ and $\operatorname{Att}_{R}(M)$ respectively. Thus Coass $(M) \subset \operatorname{Att}_{R}(M)$ and the two sets are equal when $M$ is an artinian module. The two sets behave well with respect to exact sequences. If $0 \rightarrow M^{\prime} \rightarrow M \rightarrow M^{\prime \prime} \rightarrow 0$ is an exact sequence, then

$$
\operatorname{Coass}_{R}\left(M^{\prime \prime}\right) \subset \operatorname{Coass}_{R}(M) \subset \operatorname{Coass}_{R}\left(M^{\prime}\right) \cup \operatorname{Coass}_{R}\left(M^{\prime \prime}\right)
$$

and

$$
\operatorname{Att}_{R}\left(M^{\prime \prime}\right) \subset \operatorname{Att}_{R}(M) \subset \operatorname{Att}_{R}\left(M^{\prime}\right) \cup \operatorname{Att}_{R}\left(M^{\prime \prime}\right) .
$$

There are equalities $\operatorname{Coass}_{R}\left(M \otimes_{R} N\right)=\operatorname{Coass}_{R}(M) \cap \operatorname{Supp}_{R}(N)$ and $\operatorname{Att}_{R}\left(M \otimes_{R} N\right)=\operatorname{Att}_{R}(M) \cap \operatorname{Supp}_{R}(N)$, whenever the module $N$ is required to be finite. We prove the second equality in Lemma 2.11. In particular $\operatorname{Coass}_{R}(M / \mathfrak{a} M)=\operatorname{Coass}_{R}(M) \cap \mathrm{V}(\mathfrak{a})$ and $\operatorname{Att}_{R}(M / \mathfrak{a} M)=\operatorname{Att}_{R}(M) \cap \mathrm{V}(\mathfrak{a})$ for every ideal $\mathfrak{a}$. Coassociated and attached prime ideals have been studied in particular by Zöschinger, [17] and [18].

In Corollary 2.13 we give a characterization of certain sets of coassociated primes of the highest nonvanishing local cohomology module $\mathrm{H}_{\mathfrak{a}}^{t}(M)$, where $M$ is a finitely generated module over a complete local ring. In case it happens that $t=\operatorname{dim} M$, the characterization is given in [4, Lemma 3]. In that case the 
top local cohomology module is always artinian, but in general the top local cohomology module is not artinian if $t<\operatorname{dim} M$.

\section{Main results}

First we extend a result by Zöschinger [15, Lemma 1.3] with a much weaker condition. Our method of proof is also quite different.

Proposition 2.1. Let $M$ be a module over the noetherian ring $R$. The following statements are equivalent:

(i) $M$ is a finite $R$-module.

(ii) $M_{\mathfrak{m}}$ is a finite $R_{\mathfrak{m}}$-module for all $\mathrm{m} \in \operatorname{Max} R$ and $\operatorname{Min}_{R}(M / N)$ is a finite set for all finite submodules $N \subset M$.

Proof. The only nontrivial part is (ii) $\Rightarrow$ (i).

Let $\mathscr{F}$ be the set of finite submodules of $M$. For each $N \in \mathscr{F}$ the set $\operatorname{Supp}_{R}(M / N)$ is closed in $\operatorname{Spec}(R)$, $\operatorname{since} \operatorname{Min}_{R}(M / N)$ is a finite set. Also it follows from the hypothesis that, for each $\mathfrak{p} \in \operatorname{Spec}(R)$ there is $N \in \mathscr{F}$ such that $M_{\mathfrak{p}}=N_{\mathfrak{p}}$, that is $\mathfrak{p} \notin \operatorname{Supp}_{R}(M / N)$. This means that $\bigcap_{N \in \mathscr{F}} \operatorname{Supp}_{R}(M / N)=\varnothing$. Now $\operatorname{Spec}(R)$ is a quasi-compact topological space. Consequently $\bigcap_{i=1}^{r} \operatorname{Supp}_{R}\left(M / N_{i}\right)=\varnothing$ for some $N_{1}, \ldots, N_{r} \in \mathscr{F}$. We claim that $M=N$, where $N=\sum_{i=1}^{r} N_{i}$. Just observe that $\operatorname{Supp}_{R}(M / N) \subset$ $\operatorname{Supp}_{R}\left(M / N_{i}\right)$ for each $i$, and therefore $\operatorname{Supp}_{R}(M / N)=\varnothing$.

Corollary 2.2. Let $M$ be an $R$-module such that $\operatorname{Supp} M \subset V(\mathfrak{a})$ and $M_{\mathfrak{m}}$ is $a R_{\mathfrak{m}}$-cofinite for each maximal ideal $\mathrm{m}$. The following statements are equivalent:

(i) $M$ is a-cofinite.

(ii) For all $j, \operatorname{Min}_{R}\left(\operatorname{Ext}_{R}^{j}(R / \mathfrak{a}, M) / T\right)$ is a finite set for each finite submodule $T$ of $\operatorname{Ext}_{R}^{j}(R / \mathfrak{a}, M)$.

Proof. The only nontrivial part is (ii) $\Rightarrow$ (i).

Suppose $\mathrm{m}$ is a maximal ideal of $R$. By hypothesis $M_{\mathfrak{m}}$ is $a R_{\mathfrak{m}}$-cofinite. Therefore $\operatorname{Ext}_{R}^{j}(R / \mathfrak{a}, M)_{\mathfrak{m}}$ is a finite $R_{\mathfrak{m}}$-module for all $j$. Hence by Proposition 2.1 $\operatorname{Ext}_{R}^{j}(R / \mathfrak{a}, M)$ is finite for all $j$. Thus $M$ is a-cofinite.

Corollary 2.3. Let a an ideal of $R$ such that $\operatorname{dim} R / \mathfrak{a}=1, M$ a finite $R$-module and $i \geq 0$. The following statements are equivalent:

(i) $\mathrm{H}_{\mathfrak{a}}^{i}(M)$ is a-cofinite.

(ii) For all $j, \operatorname{Min}_{R}\left(\operatorname{Ext}_{R}^{j}\left(R / \mathfrak{a}, \mathrm{H}_{\mathfrak{a}}^{i}(M)\right) / T\right)$ is a finite set for each finite submodule $T$ of $\operatorname{Ext}_{R}^{j}\left(R / \mathfrak{a}, \mathrm{H}_{\mathfrak{a}}^{i}(M)\right)$. 
Proof. For all maximal ideals $\mathfrak{m}, \mathrm{H}_{\mathfrak{a}}^{i}(M)_{\mathfrak{m}} \cong \mathrm{H}_{\mathfrak{a} R_{\mathfrak{m}}}^{i}\left(M_{\mathfrak{m}}\right)$. By [4, Theorem 1] $\mathrm{H}_{\mathfrak{a} R_{\mathfrak{l l}}}^{i}\left(M_{\mathfrak{m}}\right)$ is $\mathfrak{a} R_{\mathfrak{m}}$-cofinite.

A module $M$ is weakly Laskerian, when for each submodule $N$ of $M$ the quotient $M / N$ has just finitely many associated primes, see [6]. A module $M$ is a-weakly cofinite if $\operatorname{Supp}_{R}(M) \subset \mathrm{V}(\mathfrak{a})$ and $\operatorname{Ext}_{R}^{i}(R / \mathfrak{a}, M)$ is weakly Laskerian for all $i$. Clearly each $\mathfrak{a}$-cofinite module is $\mathfrak{a}$-weakly cofinite but the converse is not true in general see [5, Example 3.5(i) and (ii)].

Corollary 2.4. If $\mathrm{H}_{\mathfrak{a}}^{i}(M)$ (with $\operatorname{dim} R / \mathfrak{a}=1$ ) is an a-weakly cofinite module, then it is also a-cofinite.

Next we will introduce a subcategory of the category of $R$-modules that has been studied by Zöschinger in [16, Satz 1.6].

THeOREM 2.5 (Zöschinger). For any $R$-module $M$ the following are equivalent:

(i) $M$ satisfies the minimal condition for submodules $N$ such that $M / N$ is soclefree.

(ii) For any descending chain $N_{1} \supset N_{2} \supset N_{3} \supset \cdots$ of submodules of $M$, there is $n$ such that the quotients $N_{i} / N_{i+1}$ have support in Max $R$ for all $i \geq n$.

(iii) With $L(M)=\bigoplus_{\mathfrak{m} \in \operatorname{Max} R} \Gamma_{\mathfrak{m}}(M)$, the module $M / L(M)$ has finite Goldie dimension, and $\operatorname{dim} R / \mathfrak{p} \leq 1$ for all $\mathfrak{p} \in \operatorname{Ass}_{R}(M)$.

If they are fulfilled, then for each monomorphism $f: M \longrightarrow M$,

$$
\operatorname{Supp}_{R}(\operatorname{Coker} f) \subset \operatorname{Max} R \text {. }
$$

We will say that $M$ is in the class $\mathscr{Z}$ if $M$ satisfies the equivalent conditions in Theorem 2.5.

A module $M$ is soclefree if it has no simple submodules, or in other terms Ass $M \cap \operatorname{Max} R=\varnothing$. For example if $M$ is a module over the local ring $(R, \mathfrak{m})$ then the module $M / \Gamma_{\mathfrak{m}}(M)$, where $\Gamma_{\mathfrak{m}}(M)$ is the submodule of $M$ consisting of all elements of $M$ annihilated by some high power $\mathrm{m}^{n}$ of the maximal ideal $\mathfrak{m}$, is always soclefree.

Proposition 2.6. The class $\mathscr{Z}$ is a Serre subcategory of the category of $R$-modules, that is $\mathscr{Z}$ is closed under taking submodules, quotients and extensions.

Proof. The only difficult part is to show that $\mathscr{Z}$ is closed under taking extensions. To this end let $0 \longrightarrow M^{\prime} \stackrel{f}{\longrightarrow} M \stackrel{g}{\longrightarrow} M^{\prime \prime} \longrightarrow 0$ be an exact sequence with $M^{\prime}, M^{\prime \prime} \in \mathscr{Z}$ and let $N_{1} \supset N_{2} \supset \cdots$ be a descending chain of submodules of $M$. Consider the descending chains $f^{-1}\left(N_{1}\right) \supset$ 
$f^{-1}\left(N_{2}\right) \supset \cdots$ and $g\left(N_{1}\right) \supset g\left(N_{2}\right) \supset \cdots$ of submodules of $M^{\prime}$ and $M^{\prime \prime}$ respectively. By (ii) there is $n$ such that $\operatorname{Supp}_{R}\left(f^{-1}\left(N_{i}\right) / f^{-1}\left(N_{i+1}\right)\right) \subset \operatorname{Max} R$ and $\operatorname{Supp}_{R}\left(g\left(N_{i}\right) / g\left(N_{i+1}\right)\right) \subset \operatorname{Max} R$ for all $i \geq n$. We use the exact sequence

$$
0 \longrightarrow f^{-1}\left(N_{i}\right) / f^{-1}\left(N_{i+1}\right) \longrightarrow N_{i} / N_{i+1} \longrightarrow g\left(N_{i}\right) / g\left(N_{i+1}\right) \longrightarrow 0 .
$$

to conclude that $\operatorname{Supp}_{R}\left(N_{i} / N_{i+1}\right) \subset \operatorname{Max} R$ for all $i \geq n$.

THEOREM 2.7. Let $N$ be a module over a noetherian ring $R$ and $\mathfrak{a}$ an ideal of $R$ such that $\operatorname{dim} R / \mathfrak{a}=1$. If $N_{\mathfrak{m}}$ is $\mathfrak{a} R_{\mathfrak{m}}$-cofinite for all $\mathfrak{m} \in \operatorname{Max} R$, then $N$ is in the class $\mathscr{Z}$. In particular, if $M$ is a finite $R$-module, then $\mathrm{H}_{\mathfrak{a}}^{i}(M)$ is in the class $\mathscr{Z}$ for all $i$.

Proof. Let $X=N / L(N)$. Note that $\operatorname{Ass}_{R}(X) \subset \operatorname{Min} \mathfrak{a}$ and therefore is a finite set. Since

$$
\mathrm{E}(X)=\bigoplus_{\mathfrak{p} \in \operatorname{Ass}_{R}(X)} \mathrm{E}(R / \mathfrak{p})^{\mu^{i}(\mathfrak{p}, X)},
$$

it is enough to prove that $\mu^{i}(\mathfrak{p}, X)$ is finite for all $\mathfrak{p} \in \operatorname{Ass}_{R}(X)$. This is clear, since each $\mathfrak{p} \in \operatorname{Ass}_{R}(X)$ is minimal over $\mathfrak{a}$ and therefore $X_{\mathfrak{p}} \cong N_{\mathfrak{p}}$ which is, $\mathfrak{a} R_{\mathfrak{p}}$-cofinite, i.e. artinian over $R_{\mathfrak{p}}$.

Given elements $x_{1}, \ldots, x_{r}$ in $R$, we denote by $\mathrm{H}^{i}\left(x_{1}, \ldots, x_{r} ; M\right)$ the $i$ 'th Koszul cohomology module of the $R$-module $M$. The following lemma is used in the proof of Theorem 2.10.

Lemma 2.8. Let $E$ be an injective module. If $\mathrm{H}^{0}\left(x_{1}, \ldots, x_{r} ; E\right)=0$, then $\mathrm{H}^{i}\left(x_{1}, \ldots, x_{r} ; E\right)=0$ for all $i$.

Proof. We may assume that $E=\mathrm{E}(R / \mathfrak{p})$ for some prime ideal $\mathfrak{p}$, since $E$ is a direct sum of modules of this form, and Koszul cohomology preserves (arbitrary) direct sums.

Put $\mathfrak{a}=\left(x_{1}, \ldots, x_{r}\right)$. By hypothesis $0:_{E} \mathfrak{a}=0$, which means that $\mathfrak{a} \not \subset \mathfrak{p}$. Take an element $s \in \mathfrak{a} \backslash \mathfrak{p}$. It acts bijectively on $E$, hence also on $\mathrm{H}^{i}\left(x_{1}, \ldots, x_{r} ; E\right)$ for each $i$. But $a \subset \operatorname{Ann}_{R}\left(\mathrm{H}^{i}\left(x_{1}, \ldots, x_{r} ; E\right)\right)$ for all $i$, so the element $s$ therefore acts as the zero homomorphism on each $\mathrm{H}^{i}\left(x_{1}, \ldots, x_{r} ; E\right)$. The conclusion follows.

First we state the definition, given in [10], of the notion of filter regularity on modules (not necessarily finite) over any noetherian ring. When $(R, \mathfrak{m})$ is local and $M$ is finite, it yields the ordinary notion of filter-regularity, see [12].

Definition 2.9. Let $M$ be a module over the noetherian ring $R$. An element $x$ of $R$ is called filter-regular on $M$ if the module $0:_{M} x$ has finite length.

A sequence $x_{1}, \ldots, x_{s}$ is said to be filter regular on $M$ if $x_{j}$ is filter-regular on $M /\left(x_{1}, \ldots, x_{j-1}\right) M$ for $j=1, \ldots, s$. 
The following theorem yields a characterization of artinian cofinite local cohomology modules.

Theorem 2.10. Let $\mathfrak{a}=\left(x_{1}, \ldots, x_{r}\right)$ be an ideal of a noetherian ring $R$ and let $n$ be a positive integer. For each $R$-module $M$ the following conditions are equivalent:

(i) $\mathrm{H}_{\mathfrak{a}}^{i}(M)$ is artinian and $\mathfrak{a}$-cofinite for all $i<n$.

(ii) $\operatorname{Ext}_{R}^{i}(R / \mathfrak{a}, M)$ has finite length for all $i<n$.

(iii) The Koszul cohomology module $\mathrm{H}^{i}\left(x_{1}, \ldots, x_{r} ; M\right)$ has finite length for all $i<n$.

When $M$ is finite these conditions are also equivalent to:

(iv) $\mathrm{H}_{\mathfrak{a}}^{i}(M)$ is artinian for all $i<n$.

(v) There is a sequence of length $n$ in a that is filter-regular on $M$.

Proof. We use induction on $n$. When $n=1$ the conditions (ii) and (iii) both say that $0:_{M}$ a has finite length, and they are therefore equivalent to (i) [10, Proposition 4.1].

Let $n>1$ and assume that the conditions are equivalent when $n$ is replaced by $n-1$. Put $L=\Gamma_{a}(M)$ and $\bar{M}=M / L$ and form the exact sequence $0 \longrightarrow L \longrightarrow M \longrightarrow \bar{M} \longrightarrow 0$. We have $\Gamma_{\mathfrak{a}}(\bar{M})=0$ and $\mathrm{H}_{\mathfrak{a}}^{i}(\bar{M}) \cong \mathrm{H}_{\mathfrak{a}}^{i}(M)$ for all $i>0$. There are exact sequences

$$
\operatorname{Ext}_{R}^{i}(R / \mathfrak{a}, L) \rightarrow \operatorname{Ext}_{R}^{i}(R / \mathfrak{a}, M) \rightarrow \operatorname{Ext}_{R}^{i}(R / \mathfrak{a}, \bar{M}) \rightarrow \operatorname{Ext}_{R}^{i+1}(R / \mathfrak{a}, L)
$$

and

$$
\begin{aligned}
\mathrm{H}^{i}\left(x_{1}, \ldots, x_{r} ; L\right) \rightarrow \mathrm{H}^{i}\left(x_{1}, \ldots,\right. & \left.x_{r} ; M\right) \\
& \rightarrow \mathrm{H}^{i}\left(x_{1}, \ldots, x_{r} ; \bar{M}\right) \rightarrow \mathrm{H}^{i+1}\left(x_{1}, \ldots, x_{r} ; L\right)
\end{aligned}
$$

Because $L$ is artinian and $\mathfrak{a}$-cofinite the outer terms of both exact sequences have finite length. Hence $M$ satisfies one of the conditions if and only if $\bar{M}$ satisfies the same condition. We may therefore assume that $\Gamma_{\mathfrak{a}}(M)=0$.

Let $E$ be the injective hull of $M$ and put $N=E / M$. Consider the exact sequence $0 \longrightarrow M \longrightarrow E \longrightarrow N \longrightarrow 0$. We know that $0:_{M} \mathfrak{a}=0$. Therefore $0:_{E} \mathfrak{a}=0$ and $\Gamma_{\mathfrak{a}}(E)=0$. Consequently there are isomorphisms for all $i \geq 0$ :

$$
\mathrm{H}_{\mathfrak{a}}^{i+1}(M) \cong \mathrm{H}_{\mathfrak{a}}^{i}(N), \quad \operatorname{Ext}_{R}^{i+1}(R / \mathfrak{a}, M) \cong \operatorname{Ext}_{R}^{i}(R / \mathfrak{a}, N)
$$

and

$$
\mathrm{H}^{i+1}\left(x_{1}, \ldots, x_{r} ; M\right) \cong \mathrm{H}^{i}\left(x_{1}, \ldots, x_{r} ; N\right) .
$$


In order to get the third isomorphism, we used that $\mathrm{H}^{i}\left(x_{1}, \ldots, x_{r} ; E\right)=0$ for all $i \geq 0$ (Lemma 2.8). Hence $M$ satisfies one of the three conditions if and only if $N$ satisfies the same condition, with $n$ replaced by $n-1$. By induction, we may therefore conclude that the module $M$ satisfies all three conditions if it satisfies one of them.

Let now $M$ be a finite module.

(ii) $\Leftrightarrow$ (iv) Use [10, Theorem 5.5(i) $\Leftrightarrow$ (ii)].

(v) $\Rightarrow$ (i) Use [10, Theorem 6.4].

(i) $\Rightarrow$ (v) We give a proof by induction on $n$. Put $L=\Gamma_{\mathfrak{a}}(M)$ and $\bar{M}=M / L$. Then $\operatorname{Ass}_{R} L=\operatorname{Ass}_{R} M \cap \mathrm{V}(\mathfrak{a})$ and $\operatorname{Ass}_{R} \bar{M}=\operatorname{Ass}_{R} M \backslash \mathrm{V}(\mathfrak{a})$. The module $L$ has finite length and therefore $\operatorname{Ass}_{R} L \subset \operatorname{Max} R$. By prime avoidance take an element $y_{1} \in \mathfrak{a} \backslash \bigcup_{\mathfrak{p} \in \operatorname{Ass}_{R}(\bar{M})} \mathfrak{p}$. Then $\operatorname{Ass}_{R}\left(0::_{M} y_{1}\right)=\operatorname{Ass}_{R}(M) \cap \mathrm{V}\left(y_{1}\right)=$ $\left(\operatorname{Ass}_{R} L \cap \mathrm{V}\left(y_{1}\right)\right) \cup\left(\operatorname{Ass}_{R} \bar{M} \cap \mathrm{V}\left(y_{1}\right)\right) \subset \operatorname{Max} R$. Hence $0:_{M} y_{1}$ has finite length, so the element $y_{1} \in \mathfrak{a}$ is filter regular on $M$.

Suppose $n>1$ and take $y_{1}$ as above.

Note that $\mathrm{H}_{\mathfrak{a}}^{i}(M) \cong \mathrm{H}_{\mathfrak{a}}^{i}(\bar{M})$ for all $i \geq 1$. Thus we may replace $M$ by $\bar{M}$, [10, Proposition 6.3(b)], and we may assume that $y_{1}$ is a non-zerodivisor on $M$.

The exact sequence $0 \rightarrow M \stackrel{y_{1}}{\rightarrow} M \rightarrow M / y_{1} M \rightarrow 0$ yields the long exact sequence

$$
\cdots \longrightarrow \mathrm{H}_{\mathfrak{a}}^{i-1}(M) \longrightarrow \mathrm{H}_{\mathfrak{a}}^{i-1}\left(M / y_{1} M\right) \longrightarrow \mathrm{H}_{\mathfrak{a}}^{i}(M) \longrightarrow \cdots .
$$

Hence $\mathrm{H}_{\mathfrak{a}}^{i}\left(M / y_{1} M\right)$ is $\mathfrak{a}$-cofinite and artinian for all $i<n-1$, by [11, Corollary 1.7]. Therefore by the induction hypothesis there exists $y_{2}, \ldots, y_{n}$ in $\mathfrak{a}$, which is filter-regular on $M / y_{1} M$. Thus $y_{1}, \ldots, y_{n}$ is filter-regular on $M$.

REMARK. In [1] we studied the kernel and cokernel of the natural homomorphism $f: \operatorname{Ext}_{R}^{n}(R / \mathfrak{a}, M) \rightarrow \operatorname{Hom}_{R}\left(R / \mathfrak{a}, \mathrm{H}_{\alpha}^{n}(M)\right)$. Applying the criterion of Theorem 2.10 we get that if $\operatorname{Ext}_{R}^{t-j}\left(R / \mathfrak{a}, \mathrm{H}_{\mathfrak{\alpha}}^{j}(M)\right)$ has finite length for $t=n, n+1$ and for all $j<n$, then $\operatorname{Ext}_{R}^{n}(R / \mathfrak{a}, M)$ has finite length if and only if $\mathrm{H}_{\mathfrak{a}}^{n}(M)$ is $\mathfrak{a}$-cofinite artinian.

Next we will study attached and coassociated prime ideals for the last nonvanishing local cohomology module. First we prove a lemma used in Corollary 2.13

Lemma 2.11. For all $R$-modules $M$ and for every finite $R$-module $N$,

$$
\operatorname{Att}_{R}\left(M \otimes_{R} N\right)=\operatorname{Att}_{R}(M) \cap \operatorname{Supp}_{R}(N) .
$$

Proof. Let $\mathfrak{p} \in \operatorname{Att}_{R}\left(M \otimes_{R} N\right)$, so $\mathfrak{p}=\operatorname{Ann}_{R}\left(\left(M \otimes_{R} N\right) \otimes_{R} R / \mathfrak{p}\right)$. However this ideal contains both $\operatorname{Ann}_{R}(M / \mathfrak{p} M)$ and $\operatorname{Ann}_{R}(N)$ and therefore $\mathfrak{p}=\operatorname{Ann}_{R}(M / \mathfrak{p} M)$ and $\mathfrak{p} \in \operatorname{Supp}_{R}(N)$. 
Conversely let $\mathfrak{p} \in \operatorname{Att}_{R}(M) \cap \operatorname{Supp}_{R}(N)$. Then $\mathfrak{p}=\operatorname{Ann} M / \mathfrak{p} M$ and we want to show that $\mathfrak{p}=\operatorname{Ann}_{R}\left(\left(M \otimes_{R} N\right) \otimes_{R} R / \mathfrak{p}\right)$. Since

$$
\left(M \otimes_{R} N\right) \otimes_{R} R / \mathfrak{p} \cong M / \mathfrak{p} M \otimes_{R / \mathfrak{p}} N / \mathfrak{p} N,
$$

we may assume that $R$ is a domain and $\mathfrak{p}=(0)$. Let $K$ be the field of fractions of $R$. Then Ann $M=0$ and $N \otimes_{R} K \neq 0$. Therefore the natural homomorphism $f: R \longrightarrow \operatorname{End}_{R}(M)$ is injective and we have the following exact sequence

$$
0 \longrightarrow \operatorname{Hom}_{R}(N, R) \longrightarrow \operatorname{Hom}_{R}\left(N, \operatorname{End}_{R}(M)\right) \text {. }
$$

$\operatorname{But}_{\operatorname{Hom}_{R}}\left(N, \operatorname{End}_{R}(M)\right) \cong \operatorname{Hom}_{R}\left(M \otimes_{R} N, M\right)$. Hence we get $\operatorname{Ann}_{R}\left(M \otimes_{R} N\right) \subset \operatorname{Ann}_{R} \operatorname{Hom}_{R}\left(M \otimes_{R} N, M\right)$

$$
\subset \operatorname{Ann}_{R} \operatorname{Hom}_{R}(N, R) \subset \operatorname{Ann}_{R}\left(\operatorname{Hom}_{R}(N, R) \otimes_{R} K\right) .
$$

On the other hand $\operatorname{Hom}_{R}(N, R) \otimes_{R} K \cong \operatorname{Hom}_{R}\left(N \otimes_{R} K, K\right)$, which is a nonzero vector space over $K$. Consequently $\operatorname{Ann}_{R}\left(M \otimes_{R} N\right)=0$.

THEOREM 2.12. Let $(R, \mathfrak{m})$ be a complete local ring and let $\mathfrak{a}$ be an ideal of $R$. Let $t$ be a nonnegative integer such that $\mathrm{H}_{\mathfrak{a}}^{i}(R)=0$ for all $i>t$.

(a) If $\mathfrak{p} \in \operatorname{Att}_{R}\left(\mathrm{H}_{\mathfrak{a}}^{t}(R)\right)$ then $\operatorname{dim} R / \mathfrak{p} \geq t$.

(b) If $\mathfrak{p}$ is a prime ideal such that $\operatorname{dim} R / \mathfrak{p}=t$, then the following conditions are equivalent:

(i) $\mathfrak{p} \in \operatorname{Coass}_{R}\left(\mathrm{H}_{\mathfrak{a}}^{t}(R)\right)$.

(ii) $\mathfrak{p} \in \operatorname{Att}_{R}\left(\mathrm{H}_{\mathfrak{a}}^{t}(R)\right)$.

(iii) $\mathrm{H}_{\mathfrak{a}}^{t}(R / \mathfrak{p}) \neq 0$.

(iv) $\sqrt{\mathfrak{a}+\mathfrak{p}}=\mathfrak{m}$.

Proof. (a) By the right exactness of the functor $\mathrm{H}_{\mathfrak{a}}^{t}(-)$ we have

$$
\mathrm{H}_{\mathfrak{a}}^{t}(R / \mathfrak{p}) \cong \mathrm{H}_{\mathfrak{a}}^{t}(R) / \mathfrak{p} \mathrm{H}_{\mathfrak{a}}^{t}(R)
$$

If $\mathfrak{p} \in \operatorname{Att}_{R}\left(\mathrm{H}_{\mathfrak{a}}^{t}(R)\right)$, then $\mathrm{H}_{\mathfrak{a}}^{t}(R) / \mathfrak{p} \mathrm{H}_{\mathfrak{a}}^{t}(R) \neq 0$. Hence $\mathrm{H}_{\mathfrak{a}}^{t}(R / \mathfrak{p}) \neq 0$ and $\operatorname{dim} R / \mathfrak{p} \geq t$.

(b) Since $R / \mathfrak{p}$ is a complete local domain of dimension $t$, the equivalence of (iii) and (iv) follows from the local Lichtenbaum Hartshorne vanishing theorem.

If $\mathrm{H}_{\mathfrak{a}}^{t}(R / \mathfrak{p}) \neq 0$, then by (1) $\mathrm{H}_{\mathfrak{a}}^{t}(R) / \mathfrak{p} \mathrm{H}_{\mathfrak{a}}^{t}(R) \neq 0$. Therefore $\mathfrak{p} \subset \mathfrak{q}$ for some $\mathfrak{q} \in \operatorname{Coass}_{R}\left(\mathrm{H}_{\mathfrak{a}}^{t}(R)\right) \subset \operatorname{Att}_{R}\left(\mathrm{H}_{\mathfrak{a}}^{t}(R)\right)$. By (a) $\operatorname{dim} R / \mathfrak{q} \geq t=$ $\operatorname{dim} R / \mathfrak{p}$, so we must have $\mathfrak{p}=\mathfrak{q}$. Thus (iii) implies (i) and since always $\operatorname{Coass}_{R}\left(\mathrm{H}_{\mathfrak{a}}^{t}(R)\right) \subset \operatorname{Att}_{R}\left(\mathrm{H}_{\mathfrak{a}}^{t}(R)\right)$, (i) implies (ii). 
If (ii) holds then the module $\mathrm{H}_{\mathfrak{a}}^{t}(R) / \mathfrak{p} \mathrm{H}_{\mathfrak{a}}^{t}(R) \neq 0$, since its annihilator is $\mathfrak{p}$. Hence, using again the isomorphism (1), (ii) implies (iii).

Corollary 2.13. Let $(R, \mathrm{~m})$ be a complete local ring, a an ideal of $R$ and $M$ a finite R-module and $t$ a nonnegative integer such that $\mathrm{H}_{\mathfrak{a}}^{i}(M)=0$ for all $i>t$.

(a) If $\mathfrak{p} \in \operatorname{Att}_{R}\left(\mathrm{H}_{\mathfrak{a}}^{t}(M)\right)$ then $\operatorname{dim} R / \mathfrak{p} \geq t$.

(b) If $\mathfrak{p}$ is a prime ideal in $\operatorname{Supp}_{R}(M)$ such that $\operatorname{dim} R / \mathfrak{p}=t$, then the following conditions are equivalent:

(i) $\mathfrak{p} \in \operatorname{Coass}_{R}\left(\mathrm{H}_{\mathfrak{a}}^{t}(M)\right)$.

(ii) $\mathfrak{p} \in \operatorname{Att}_{R}\left(\mathrm{H}_{\mathfrak{a}}^{t}(M)\right)$.

(iii) $\mathrm{H}_{\mathfrak{a}}^{t}(R / \mathfrak{p}) \neq 0$.

(iv) $\sqrt{\mathfrak{a}+\mathfrak{p}}=\mathfrak{m}$.

Proof. Passing from $R$ to $R /$ Ann $M$, we may assume that Ann $M=0$ and therefore using Gruson's theorem, see [13, Theorem 4.1], $\mathrm{H}_{\mathfrak{a}}^{i}(N)=0$ for all $i>t$ and every $R$-module $N$. Hence the functor $\mathrm{H}_{\mathfrak{a}}^{t}(-)$ is right exact and therefore, since it preserves direct limits, we get

$$
\mathrm{H}_{\mathfrak{a}}^{t}(M) \cong M \otimes_{R} \mathrm{H}_{\mathfrak{a}}^{t}(R) .
$$

The claims follow from Theorem 2.12 using the following equalities

$$
\operatorname{Coass}_{R}\left(\mathrm{H}_{\mathfrak{a}}^{t}(M)\right)=\operatorname{Coass}_{R}\left(\mathrm{H}_{\mathfrak{a}}^{t}(R)\right) \cap \operatorname{Supp}_{R}(M)
$$

by [16, Folgerung 3.2] and

$$
\operatorname{Att}_{R}\left(\mathrm{H}_{\mathfrak{a}}^{t}(M)\right)=\operatorname{Att}_{R}\left(\mathrm{H}_{\mathfrak{a}}^{t}(R)\right) \cap \operatorname{Supp}_{R}(M)
$$

by Lemma 2.11 .

\section{REFERENCES}

1. Aghapournahr, M., Melkersson, L., A natural map in local cohomology, preprint.

2. Brodmann, M. P., Sharp, R. Y., Local Cohomology: an Algebraic Introduction with Geometric Applications, Cambridge Studies on Advanced Math. 60, Cambridge University Press, Cambridge 1998.

3. Bruns, W., Herzog, J., Cohen-Macaulay Rings, revised ed., Cambridge Studies on Advanced Math. 39, Cambridge University Press, Cambridge 1998.

4. Delfino, D., and Marley, T., Cofinite modules and local cohomology, J. Pure Appl. Algebra 121 (1997), 45-52.

5. Divaani-Aazar, K., Mafi, A., Associated primes of local cohomology modules of weakly Laskerian modules, Comm. Algebra 34 (2006), 681-690. 
6. Divaani-Aazar, K., Mafi, A., Associated primes of local cohomology modules, Proc. Amer. Math. Soc. 133 (2005), 655-660.

7. Grothendieck, A., Cohomologie locale des faisceaux cohérents et théorèmes de Lefschetz. locaux et globaux (SGA 2), North-Holland, Amsterdam 1968.

8. Hartshorne, R., Affine duality and cofiniteness, Invent. Math. 9 (1970), 145-164.

9. Matsumura, H., Commutative Ring Theory, Cambridge Studies on Advanced Math. 8, Cambridge University Press, Cambridge 1986.

10. Melkersson, L., Modules cofinite with respect to an ideal, J. Algebra 285 (2005), 649-668.

11. Melkersson, L., Properties of cofinite modules and applications to local cohomology, Math. Proc. Cambridge Phil. Soc. 125 (1999), 417-423.

12. Schenzel, P., Trung, N. V., Cuong, N. T., Verallgemeinerte Cohen-Macaulay-Moduln, Math. Nachr. 85 (1978), 57-73.

13. Vasconcelos, W., Divisor Theory in Module Categories, North-Holland Math. Studies 14, North-Holland, Amsterdam 1974.

14. Yoshida, K. I., Cofiniteness of local cohomology modules for ideals of dimension one, Nagoya Math. J. 147 (1997), 179-191.

15. Zöschinger, H., Koatomare Moduln, Math. Z. 170 (1980), 221-232.

16. Zöschinger, H., Minimax-Moduln, J. Algebra 102 (1986), 1-32.

17. Zöschinger, H., Über koassoziierte Primideale, Math Scand. 63 (1988), 196-211.

18. Zöschinger, H., Linear-kompakte Moduln über noetherschen Ringen, Arch. Math. 41 (1983), $121-130$.

ARAK UNIVERSITY

BEHESHTI ST

P.O. BOX: 879

ARAK

IRAN

E-mail: m-aghapour@araku.ac.ir

m.aghapour@gmail.com
DEPARTMENT OF MATHEMATICS

LINKÖPING UNIVERSITY

SE-581 83 LINKÖPING

SWEDEN

E-mail: lemel@mai.liu.se 